\title{
Viscous Flows Driven by Uniform Shear over a Porous Stretching Sheet in the Presence of Suction/Blowing
}

\author{
Samir Kumar Nandy ${ }^{1}$ and Swati Mukhopadhyay ${ }^{2}$ \\ ${ }^{1}$ Department of Mathematics, A.K.P.C Mahavidyalaya, Bengai, Hooghly 712 611, India \\ ${ }^{2}$ Department of Mathematics, The University of Burdwan, West Bengal 713104, India \\ Correspondence should be addressed to Samir Kumar Nandy; nandysamir@yahoo.com
}

Received 21 February 2014; Revised 10 May 2014; Accepted 12 May 2014; Published 25 May 2014

Academic Editor: Boming Yu

Copyright (c) 2014 S. K. Nandy and S. Mukhopadhyay. This is an open access article distributed under the Creative Commons Attribution License, which permits unrestricted use, distribution, and reproduction in any medium, provided the original work is properly cited.

\begin{abstract}
An analysis is carried out to study the steady two-dimensional flow of an incompressible viscous fluid past a porous deformable sheet, which is stretched in its own plane with a velocity proportional to the distance from the fixed point subject to uniform suction or blowing. A uniform shear flow of strain rate $\beta$ is considered over the stretching sheet. The analysis of the result obtained shows that the magnitude of the wall shear stress increases with the increase of suction velocity and decreases with the increase of blowing velocity and this effect is more pronounced for suction than blowing. It is seen that the horizontal velocity component (at a fixed streamwise position along the plate) increases with the increase in the ratio of shear rate $\beta$ and stretching rate (c) (i.e., $\beta / c)$ and there is an indication of flow reversal. It is also observed that this flow reversal region increases with the increase in $\beta / c$.
\end{abstract}

\section{Introduction}

Suction or injection (blowing) of a fluid through the bounding surface can significantly change the flow field and consequently affects the heat transfer rate from the surface. Injection or withdrawal of fluid through a porous bounding heated/cooled wall is of great general interest in practical problems such as cooling of films, cooling of wires, and coating of polymer fiber. The process of suction or blowing has its importance in many engineering and industrial activities such as in the design of thrust bearing and radial diffusers and thermal oil recovery. Suction is also applied to chemical processes to remove reactants whereas blowing is used to add reactants, prevent corrosion or scaling, and reduce the drag.

During the last decades, the study of flow over a stretching surface has attracted much more interest of the researchers due to its various industrial applications such as extrusion of polymer sheets, continuous stretching, manufacturing plastic films, and artificial fibers. In a melt-spinning process, the extrudate from the die is generally drawn and simultaneously stretched into a sheet which is then solidified through quenching or gradual cooling by direct contact with the water. The mechanical properties of the final product depend crucially on the rate of cooling/heating along the surface while being stretched. Sakiadas [1] was the first to study boundary layer flow due to a rigid flat continuous surface moving in its own plane. Erickson et al. [2] analyzed the boundary layer flow due to the motion of a porous flat plate when the transverse velocity at the surface is nonzero. A detailed analysis of the boundary layer flow due to a stretching sheet has been carried out by Danberg and Fansler [3].

Later, Crane [4] gave an exact similarity solution in closed analytical form for steady boundary layer flow of an incompressible viscous fluid caused solely by the stretching of an elastic flat sheet which moves in its own plane with a velocity varying linearly with distance from a fixed point. Wang [5] investigated a uniform shear flows over a quiescent liquid and he showed that an interfacial boundary layer develops both in the air and the liquid. P. S. Gupta and A. S. Gupta [6] investigated the heat and mass transfer corresponding to the similarity solution for the boundary layer flow over a stretching sheet subject to uniform suction or blowing. Rajagopal et al. [7] studied the boundary layer 
flow over a stretching surface for second-order fluid and obtained similarity solutions of the boundary layer equations. Dandapat and Gupta [8] extended the same problem with heat transfer and found exact analytical solutions.

Shear driven flows, namely, wall driven Couette flow, wind driven Ekman flow, and so forth are the classical topics of fluid mechanics. Due to their wide range of applications shear driven flows attract the attention of the researchers. The study of boundary layer flow driven by uniform shear is seen to have fewer contributors in fluid mechanics. Rajagopal et al. [9] studied the nonsimilar boundary layer flow of a second-order fluid over a stretching sheet in the presence of a uniform shear flow. Weidman et al. [10] reported a similarity solution of the boundary layer flow over a flat impermeable plate with free shear flows driven by rotational velocities. The extension of the same problem to a permeable flat plate was analyzed by Magyari et al. [11]. Cossali [12] gave the similarity solutions of the energy and momentum boundary layer equations for a power-law shear driven flow over a semi-infinite flat plate. The thermal boundary layer beneath an external uniform shear flow was considered by Magyari and Weidman [13]. Weidman et al. [14] also investigated the effects of plate extension and transpiration on uniform shear flow over a semi-infinite flat plate by considering the boundary layer approximations. Due to the presence of shear in the free stream, the free stream is no longer rotation-free. As a result the flow behaviours are quite different from the rotation-free flow. In most of the works the flat surface was kept stationary. But the interaction of shear flow and wall stretching affects the flow significantly. Fang [15] analyzed the heat transfer characteristics for boundary layer flow past a stretching sheet in presence of uniform shear-free stream. $\mathrm{Xu}$ [16] obtained the analytic solution in case of boundary layer flow driven by power-law shear. Very recently, the heat transfer characteristics of a viscous incompressible fluid over a stretching/shrinking sheet in a uniform shear flow with a convective surface boundary condition were analyzed by Aman et al. [17].

Motivated by the above investigations, in this paper, the steady, two-dimensional incompressible viscous fluid flow past a porous stretching sheet in presence of uniform suction/blowing has been investigated. A uniform shear flow of strain rate $\beta$ is considered over the stretching surface. The behaviours of the horizontal component of the flow velocity are explained and it is seen that there is a flow reversal in case of suction or blowing at the sheet.

\section{Flow Analysis}

Consider the steady two-dimensional flow of a viscous fluid towards a stretching surface coinciding with the plane $y=0$, the flow being confined to the region $y>0$. We choose the coordinate system such that the $x$-axis is along the sheet, $y$ axis is normal to the sheet, and the origin of the coordinate system is located at a finite position on the sheet. Two equal and opposing forces are applied on the stretching surface along the $x$-axis so that the surface is stretched keeping the origin fixed. Here we consider a uniform shear flow of strain rate $\beta$ over the stretching surface. The free stream velocity is

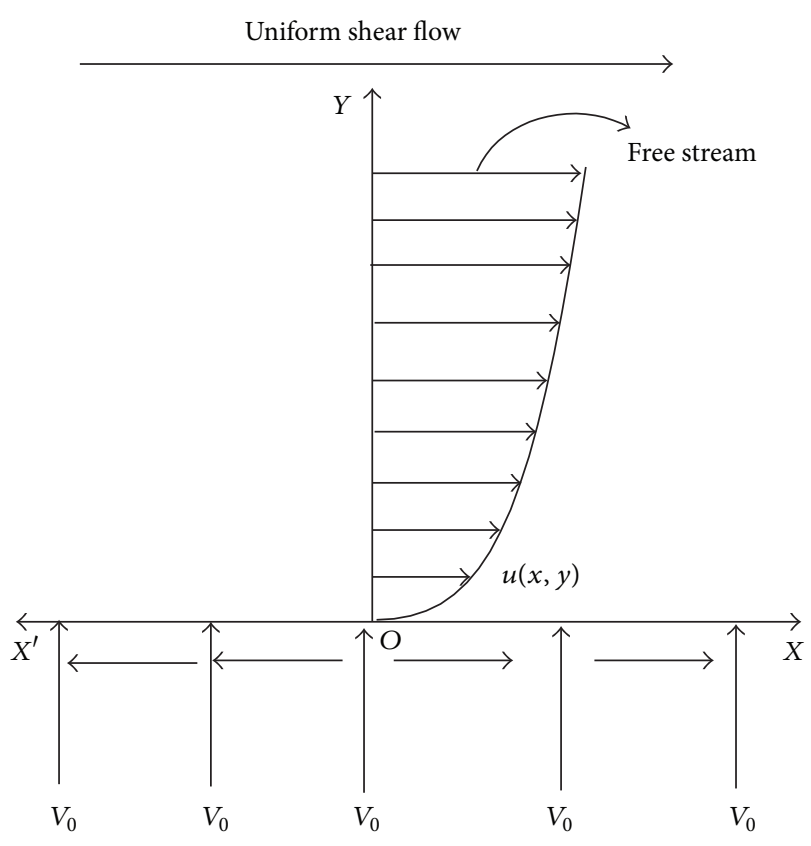

FIgURE 1: A sketch of the physical problem and the coordinate system involved.

uniform shear and the surface is subject to uniform suction or blowing. It is to be noted that, due to the presence of blowing or suction, a streamwise pressure gradient is required to maintain the flow. The flow configuration is shown in Figure 1.

The inviscid version of the present steady flow is given in terms of the stream function $\psi_{0}$ as

$$
\psi_{0}=\beta y\left(\frac{y}{2}-\delta_{1}\right)+\delta_{2} x
$$

where $\delta_{1}$ and $\delta_{2}$ are two constants. Here $\delta_{1}$ is the displacement thickness arising out of the boundary layer on the stretching surface and $\delta_{2}$ is the parameter which controls the horizontal pressure gradient that produces the shear flow. So the parameters $\delta_{1}$ and $\delta_{2}$ have some effects on the flow field (see Drazin and Riley [18]). The velocity components along $x$ and $y$ directions, respectively, for the flow described by (1) are

$$
U_{1}=\beta\left(y-\delta_{1}\right), \quad V_{1}=-\delta_{2} .
$$

The boundary conditions at the stretching surface are

$$
u=c x, \quad v=v_{w} \quad \text { at } y=0,
$$

where $c>0$ is a constant. The boundary conditions at infinity are

$$
u \longrightarrow U_{1}(x, y), \quad v \longrightarrow V_{1}(x, y) \text { as } y \longrightarrow \infty,
$$

where $U_{1}$ and $V_{1}$ are given by (2). Near the stretching surface, we take the stream function in the form:

$$
\frac{\psi}{\nu}=\xi F(\eta)+W(\eta)
$$


where

$$
\xi=\left(\frac{c}{v}\right)^{1 / 2} x, \quad \eta=\left(\frac{c}{v}\right)^{1 / 2} y,
$$

and $v$ is the kinematic viscosity. The velocity components $u$ and $v$, along $x$ and $y$ directions, are given by

$$
u=\frac{\partial \psi}{\partial y}, \quad v=-\frac{\partial \psi}{\partial x} .
$$

Hence the dimensionless velocity components $U$ and $V$ are obtained from (5) and (6) as

$$
\begin{aligned}
& U=\xi F^{\prime}(\eta)+W^{\prime}(\eta) \\
& V=-F(\eta),
\end{aligned}
$$

where $U=u /(c v)^{1 / 2}$ and $V=v /(c v)^{1 / 2}$. Substituting (8) into the Navier-Stokes equations we get

$$
\begin{aligned}
-\frac{1}{\rho} \frac{\partial p}{\partial x}= & \nu^{2}\left(\frac{c}{v}\right)^{3 / 2} \\
& \times\left[\xi\left(F^{\prime 2}-F F^{\prime \prime}-F^{\prime \prime \prime}\right)+\left(F^{\prime} W^{\prime}-F W^{\prime \prime}-W^{\prime \prime \prime}\right)\right] \\
& \quad-\frac{1}{\rho} \frac{\partial p}{\partial y}=v^{2}\left(\frac{c}{v}\right)^{3 / 2}\left[F F^{\prime}+F^{\prime \prime}\right],
\end{aligned}
$$

where a prime denotes differentiation with respect to $\eta$. Eliminating $p$ between (9) and equating the coefficients of $\xi^{0}$ and $\xi^{1}$, we get upon integration

$$
\begin{gathered}
F^{2}-F F^{\prime \prime}-F^{\prime \prime \prime}=C_{1}, \\
F^{\prime} W^{\prime}-F W^{\prime \prime}-W^{\prime \prime \prime}=C_{2},
\end{gathered}
$$

where $C_{1}$ and $C_{2}$ are the constants of integration.

The boundary conditions ( 3 ) and (4) become

$$
\begin{gathered}
F(0)=-V_{0}, \quad F^{\prime}(0)=1, \quad F^{\prime}(\infty)=0, \\
W(0)=0, \quad W^{\prime}(0)=0, \\
W^{\prime}(\eta)=\frac{\beta}{c}\left(\eta-d_{1}\right) \quad \text { as } \eta \longrightarrow \infty,
\end{gathered}
$$

where $V_{0}=v_{w} /(c v)^{1 / 2}$ and $d_{1}=(c / v)^{1 / 2} \delta_{1}$. It is to be noted that the boundary condition $W(0)=0$ is taken due to the steam function. Let us assume that $F(\eta) \rightarrow d_{2}$ as $\eta \rightarrow \infty$, where $d_{2}=\delta_{2} /(c v)^{1 / 2}$. Here $d_{2}$ is the dimensionless shear rate parameter linked to the shear flow and $d_{1}$ is the dimensionless displacement thickness parameter. The constants $C_{1}$ and $C_{2}$ are now obtained from (10) using (11) and (12) as $C_{1}=0$ and $C_{2}=-(\beta / c) d_{2}$. Hence (10) becomes

$$
\begin{aligned}
F^{2}-F F^{\prime \prime}-F^{\prime \prime \prime} & =0, \\
F^{\prime} W^{\prime}-F W^{\prime \prime}-W^{\prime \prime \prime} & =-\frac{\beta}{c} d_{2} .
\end{aligned}
$$

Here the dimensionless shear rate parameter $d_{2}$ is obtained by integrating (13) numerically by using the boundary condition (11). When (13) and (14) are substituted into (9), we get the dimensionless pressure distribution $P(\xi, \eta)$ after integration as

$$
-P=\frac{1}{2} F^{2}+F^{\prime}-\frac{\beta}{c} d_{2} \xi+\text { constant, }
$$

where $P=p(x, y) /(\rho c v)$. The dimensionless wall shear stress $T$ is given as

$$
T=\xi F^{\prime \prime}(0)+W^{\prime \prime}(0) .
$$

The dimensionless stream function for the flow can be obtained as

$$
\psi(\xi, \eta)=\xi F(\eta)+\int_{0}^{\eta} W^{\prime}(\eta) d \eta,
$$

where $\xi$ is the dimensionless distance along the surface.

\section{Method of Numerical Solution}

In the absence of an analytic solution of a problem, a numerical solution is indeed an obvious and natural choice. Thus the governing equations (13) and (14) along with the boundary conditions (11)-(12) are solved numerically by fourth-order Runge-Kutta method with shooting technique. To do this, we first transform the nonlinear differential equation (13) to a system of three first-order differential equations as

$$
y_{1}^{\prime}=y_{2}, \quad y_{2}^{\prime}=y_{3}, \quad y_{3}^{\prime}=y_{2}^{2}-y_{1} y_{3},
$$

where $y_{1}=F(\eta), y_{2}=F^{\prime}(\eta), y_{3}=F^{\prime \prime}(\eta)$, and a prime denotes differentiation with respect to the independent variable $\eta$. The boundary conditions (11) become

$$
\begin{gathered}
y_{1}=-V_{0}, \quad y_{2}=1 \quad \text { at } \eta=0, \\
y_{2} \longrightarrow 0 \quad \text { as } \eta \longrightarrow \infty .
\end{gathered}
$$

For a given value of $V_{0}$, the values of $y_{1}$ and $y_{2}$ are known at the starting point $\eta=0$. Now the value of $y_{2}$ as $\eta \rightarrow \infty$ is replaced by $y_{2}$ at a finite value $\eta=\eta_{\infty}$ to be determined later. The value of $y_{2}$ at $\eta=0$ is guessed in order to initiate the integration scheme. Starting from the given values of $y_{1}$ and $y_{2}$ at $\eta=0$ and the guessed value of $y_{3}$ at $\eta=0$, we integrate the system of first-order equations (18) by using a fourthorder Runge-Kutta method up to the end-point $\eta=\eta_{\infty}$. The computed value of $y_{2}$ at $\eta=\eta_{\infty}$ is then compared with $y_{2}$ at $\eta=\eta_{\infty}$. The absolute difference between these two values should be as small as possible. To this end we use a NewtonRaphson iteration procedure to assure quadratic convergence of the iterations. The value of $\eta_{\infty}$ is then increased till $y_{2}$ attains the value zero asymptotically.

Using the numerical values of $F(\eta)$ obtained from the solutions of (11) and (13), (14) along with the boundary conditions (12) is solved numerically using the same method as described above to obtain $W(\eta)$. 


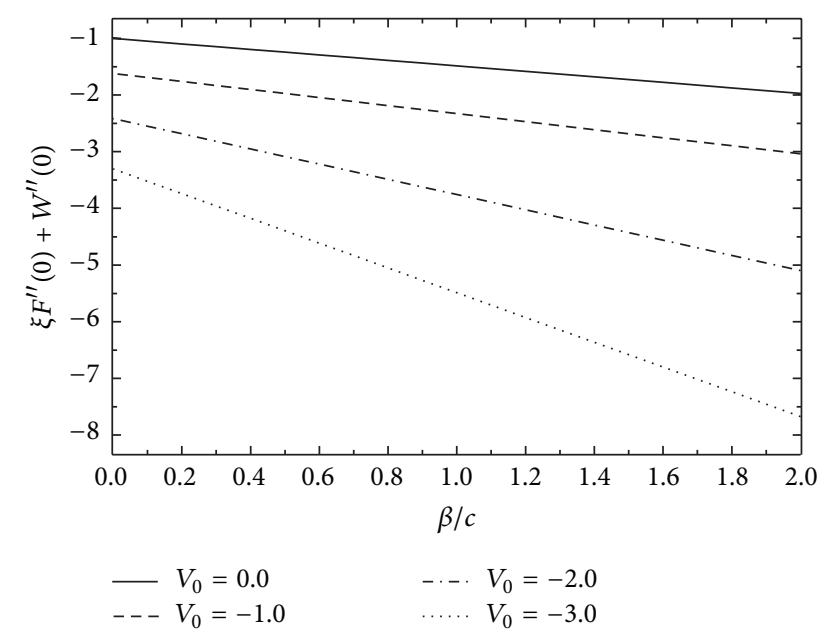

FIGURE 2: Variation of wall shear stress with $\beta / c$ for several values of $V_{0}(\leq 0)$ (i.e., suction at the plate) when $\xi=1.0$ and $d_{1}=1.0$.

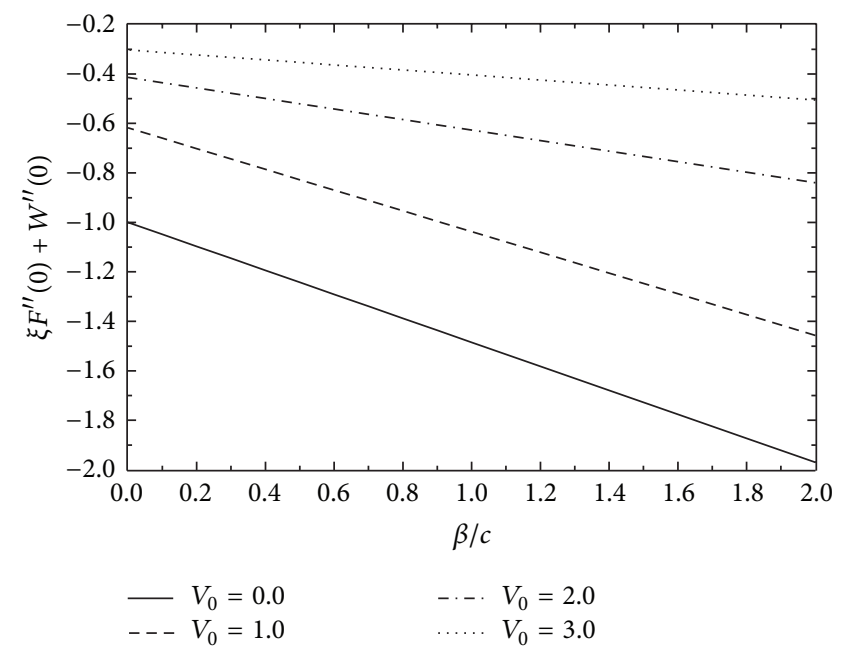

FIGURE 3: Variation of wall shear stress with $\beta / c$ for several values of $V_{0}(\geq 0)$ (i.e., blowing at the plate) when $\xi=1.0$ and $d_{1}=1.0$.

\section{Results and Discussion}

Figures 2 and 3 show the variation of dimensionless wall shear stress with $\beta$ for several values of $V_{0}$ keeping the values of the other parameters fixed. Note that $V_{0}=0$ corresponds to the case when there is no suction or blowing at the sheet, $V_{0}<0$ corresponds to suction, and $V_{0}>0$ corresponds to blowing at the sheet. Figure 2 reveals that as the suction velocity increases, the magnitude of the wall shear stress increases. On the other hand, Figure 3 indicates that with the increase of blowing velocity at the plate, the magnitude of the wall shear stress decreases. From these figures, it is also noticed that the effect of wall shear stress is more pronounced for suction than blowing at the stretching sheet.

Figure 4 shows the variation of $U(\xi, \eta)$, the horizontal component of velocity, with $\eta$ for several values of $\beta / c$ when there is no suction or blowing velocity at the sheet. Figure 5 shows the same behaviour for suction $\left(V_{0}=-2.0\right)$ at the

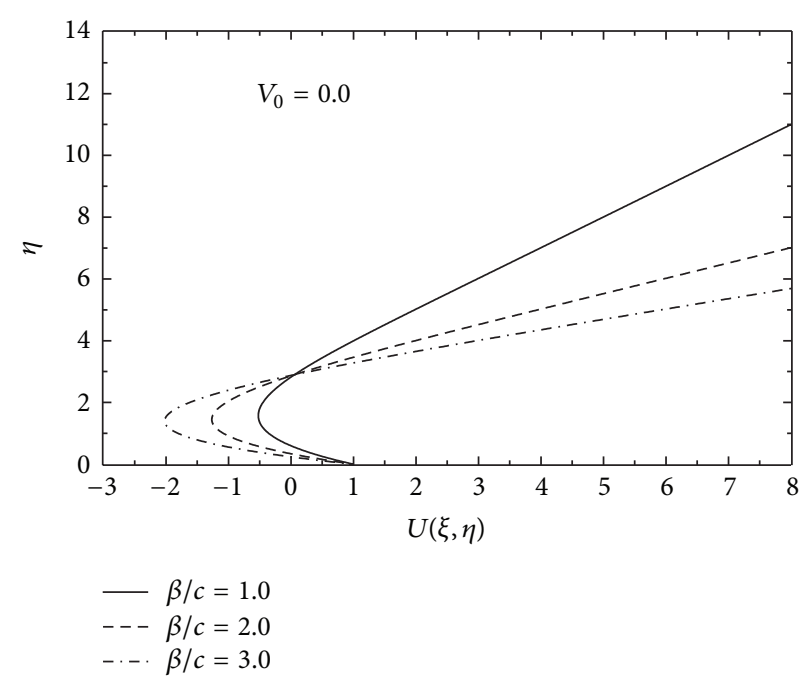

FIGURE 4: Variation of $U(\xi, \eta)$ with $\eta$ for several values of $\beta / c$ with $\xi=1.0, d_{1}=3.0$, and $V_{0}=0$ (i.e., no suction or blowing at the plate).

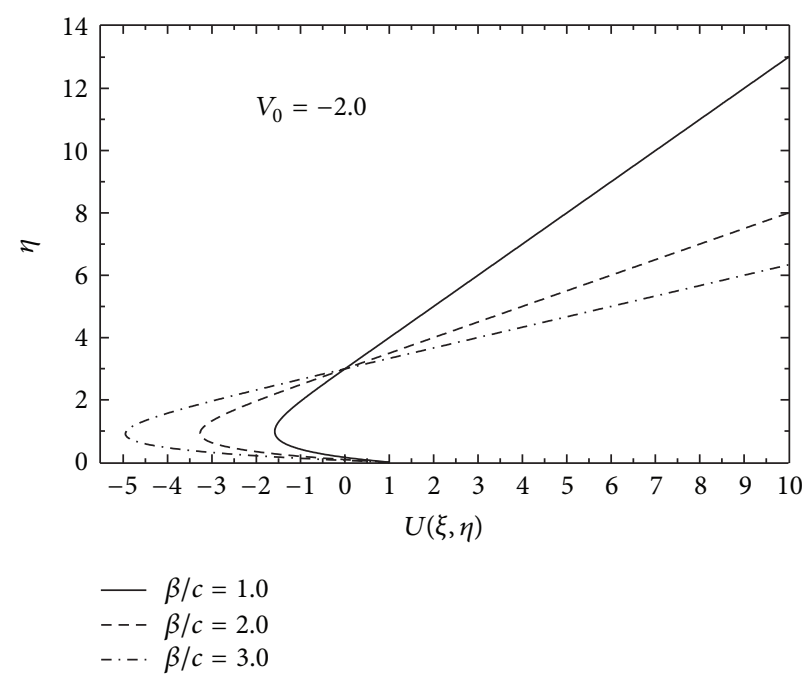

FigURE 5: Variation of $U(\xi, \eta)$ with $\eta$ for several values of $\beta / c$ with $\xi=1.0, d_{1}=3.0$, and $V_{0}=-2.0$ (i.e., for suction at the plate).

stretching sheet and Figure 6 shows for blowing $\left(V_{0}=2.0\right)$ at the stretching sheet keeping other parameters fixed. From these figures it is observed that $U(\xi, \eta)$ increases with the increase of $\beta / c$ except in a small region near the sheet. The figures indicate that there is a flow reversal very near the sheet. This region of flow reversal gradually increases with the increase of $\beta / c$.

Figures 7 and 8 show the variation of $U(\xi, \eta)$ with $\eta$ for several values of the suction and blowing at the plate, respectively. Figure 7 reveals that as $\left|V_{0}\right|$ increases (i.e., suction velocity increases), the horizontal velocity at a point decreases except in a small region near the sheet. Figure 8 shows that as the blowing velocity increases, velocity at a point increases. Notice that there is an indication of flow reversal. From these two figures, it can also be concluded that, 


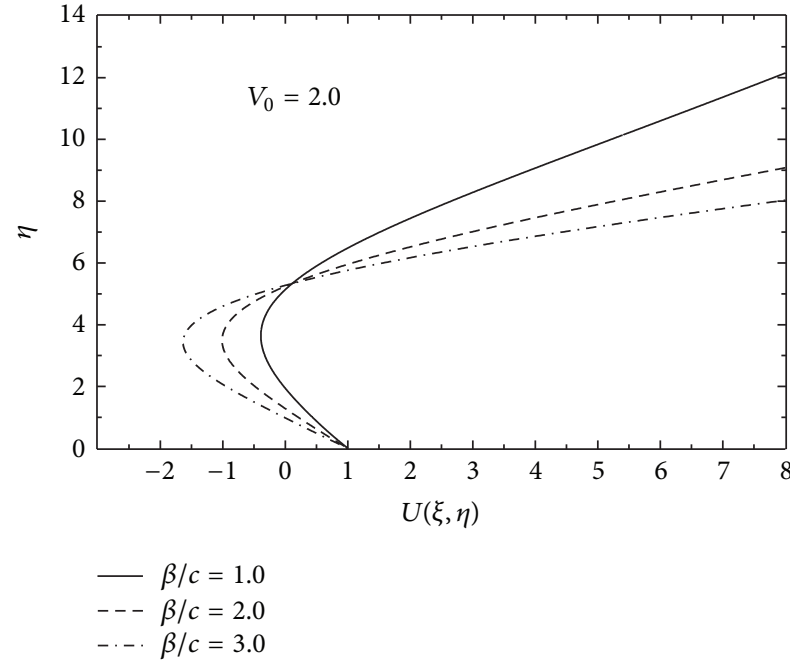

FIGURE 6: Variation of $U(\xi, \eta)$ with $\eta$ for several values of $\beta / c$ with $\xi=1.0, d_{1}=3.0$, and $V_{0}=2.0$ (i.e., for blowing at the plate).

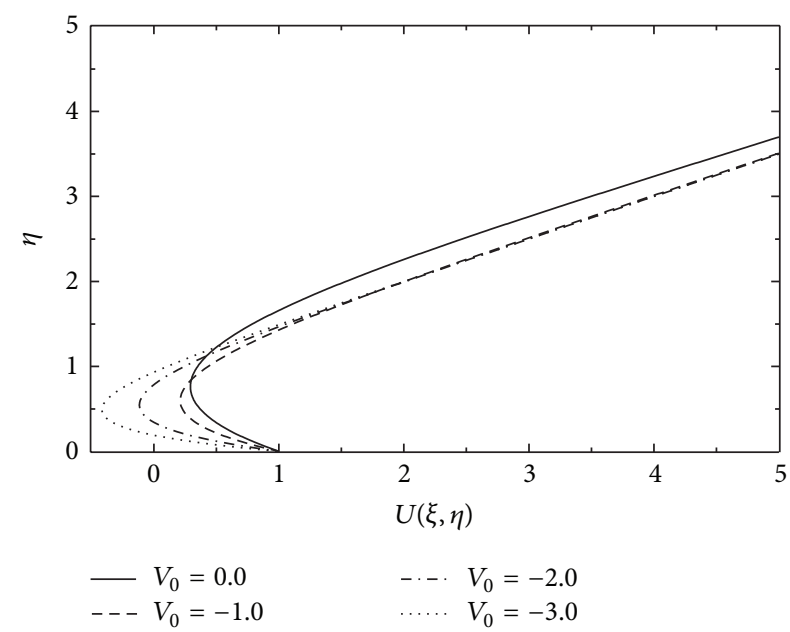

Figure 7: Variation of $U(\xi, \eta)$ with $\eta$ for several values of $V_{0}(\leq 0)$ (i.e., suction at the plate) when $\beta / c=2.0, \xi=1.0$, and $d_{1}=1.0$.

with the increase of $V_{0}$ (magnitude), the flow reversal region increases.

Figure 9 shows the variation of $U(\xi, \eta)$ with $\eta$ for several values of the shear rate parameter $d_{1}$ in the absence of suction or blowing at the sheet. Figure 10 shows the same for suction and Figure 11 stands for blowing at the sheet for the same set of parameters. From these figures it is seen that, with the increase of shear rate parameter $d_{1}$, the region of flow reversal increases. The tendency of flow reversal is more prominent in case of suction than blowing. It is also observed that as the shear rate parameter $d_{1}$ increases, velocity at a point also increases. Figure 12 depicts the variation of the horizontal component of velocity, $U(\xi, \eta)$, with $\eta$ for several values of $\xi$ (negative, zero, and positive) in the presence of blowing at the sheet. The figure reveals that as $\xi$ increases, velocity at a point decreases.

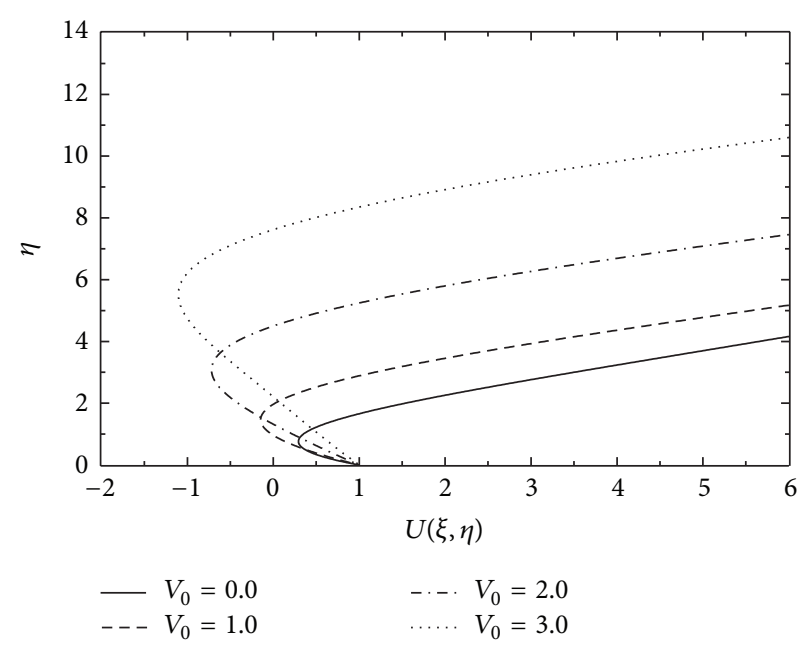

Figure 8: Variation of $U(\xi, \eta)$ with $\eta$ for several values of $V_{0}(\geq 0)$ (i.e., blowing at the plate) when $\beta / c=2.0, \xi=1.0$, and $d_{1}=1.0$.

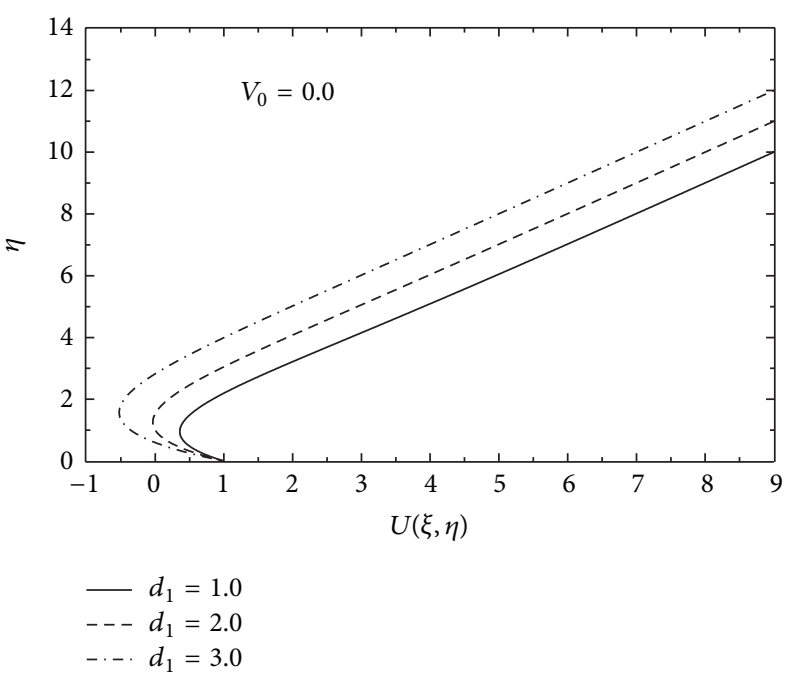

FIGURE 9: Variation of $U(\xi, \eta)$ with $\eta$ for several values of $d_{1}$ with $\xi=1.0, \beta / c=2.0$, and $V_{0}=0$ (i.e., no suction or blowing at the plate).

Presentation of full stability analysis is beyond the scope of the present work since a stability analysis requires an unsteady flow, whereas our problem is a steady one. But as we know that, in boundary layer flow, the reverse profiles suggest temporal instability so one can expect that, in the case of full Navier-Stokes solution, the same feature holds good. This can be verified using the stability analysis by adopting the techniques of Merkin [19] and Mahapatra et al. [20].

\section{Concluding Remark}

We have investigated the steady two-dimensional flow of incompressible viscous fluid past a porous deformable sheet which is stretched in its own plane with velocity $c x, x$ being the distance along the sheet from the fixed point with uniform suction or blowing. A uniform shear flow of 


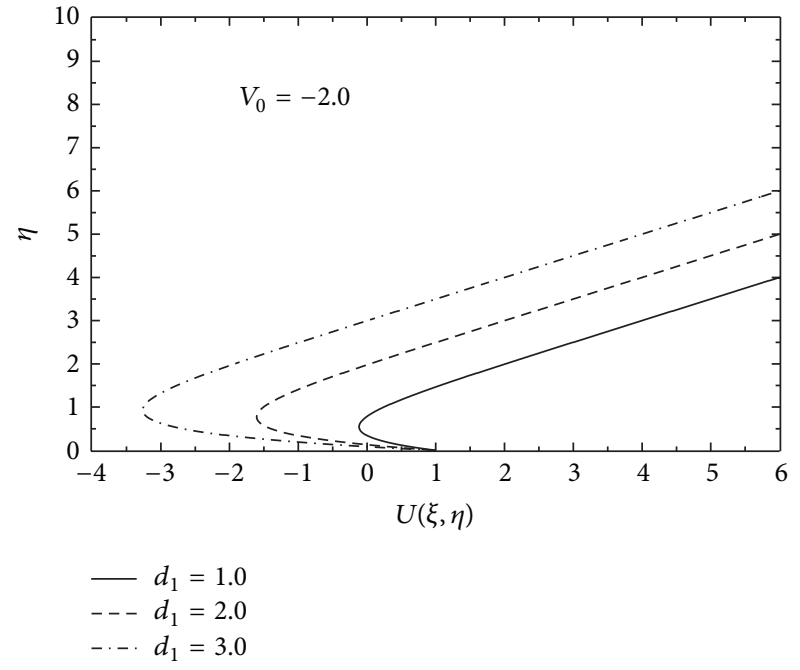

FigURE 10: Variation of $U(\xi, \eta)$ with $\eta$ for several values of $d_{1}$ with $\xi=1.0, \beta / c=2.0$, and $V_{0}=-2.0$ (i.e., for suction at the plate).

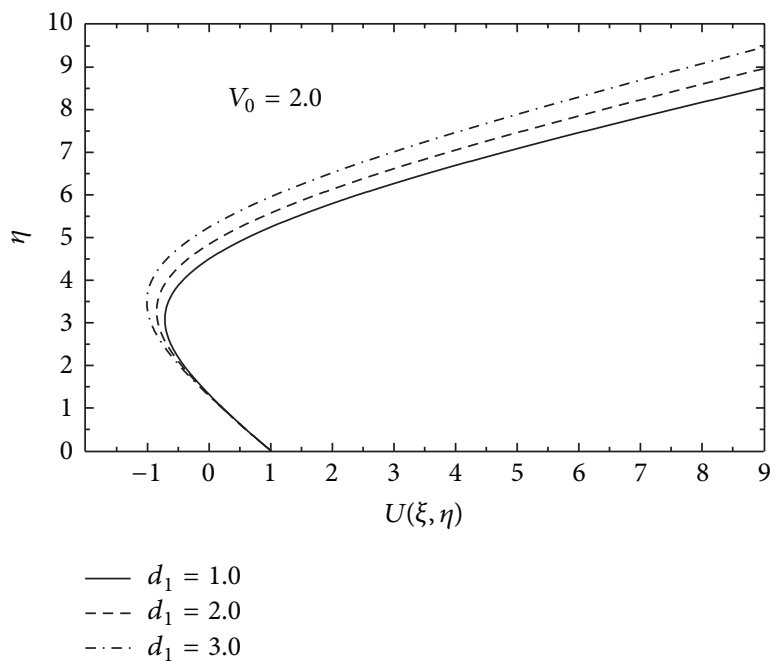

FIGURE 11: Variation of $U(\xi, \eta)$ with $\eta$ for several values of $d_{1}$ with $\xi=1.0, \beta / c=2.0$, and $V_{0}=2.0$ (i.e., for blowing at the plate).

strain rate $\beta$ is considered here over the stretching surface. It is seen that the magnitude of wall skin friction increases with the increase of suction velocity (magnitude) but it decreases with the increase of blowing velocity and this effect is more pronounced in suction than blowing. The horizontal component of velocity at a fixed point increases with the increase of $\beta / c$ and there is a flow reversal. This region of flow reversal increases with the increase of $\beta / c$. The behaviour of the horizontal velocity component is shown for different parameters.

\section{Conflict of Interests}

The authors declare that there is no conflict of interests regarding the publication of this paper.

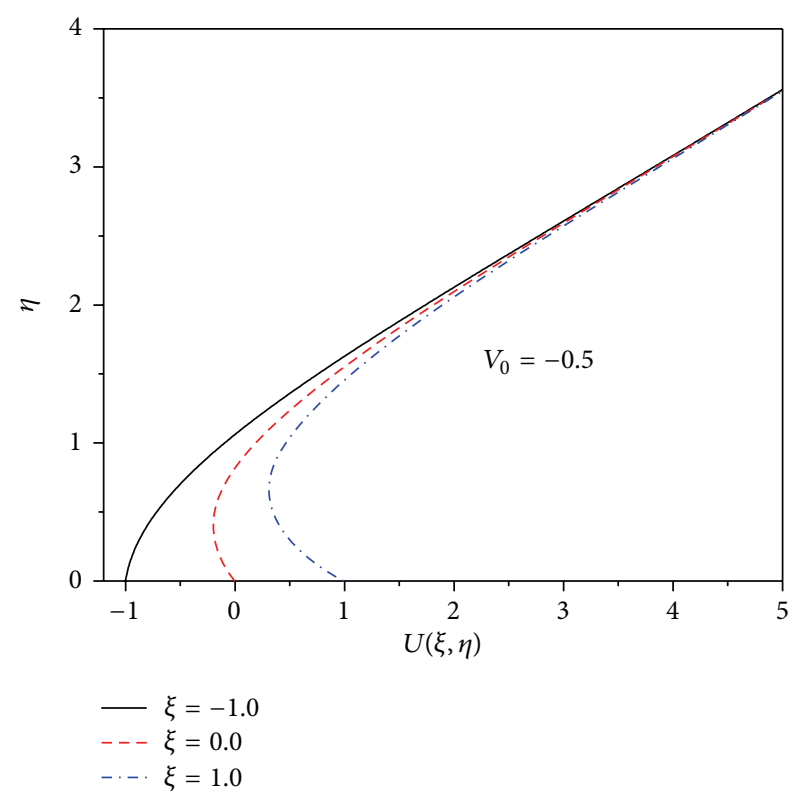

Figure 12: Variation of $U(\xi, \eta)$ with $\eta$ for several values of $\xi$ with $d_{1}=1.0, \beta / c=2.0$, and $V_{0}=-0.5$ (i.e., for suction at the plate).

\section{Acknowledgments}

The author Samir Kumar Nandy is grateful to the University Grants Commission, New Delhi, India, for providing financial support under a minor research project Grant no. F. PSW-002/13-14 to carry out the work. Authors would like to thank referees for the valuable comments which enhanced the quality of the paper.

\section{References}

[1] B. C. Sakiadas, "Boundary layer behaviour on continuous solid surface," AIChE Journal, vol. 7, pp. 26-28, 1961.

[2] L. E. Erickson, L. T. Fan, and V. G. Fox, "Heat and mass transfer on a moving continuous flat plate with suction or injection," Industrial and Engineering Chemistry Fundamentals, vol. 5, no. 1, pp. 19-25, 1966.

[3] J. E. Danberg and K. S. Fansler, "A non-similar moving-wall boundary-layer problem," Quarterly of Applied Mathematics, vol. 34, pp. 305-309, 1976.

[4] L. J. Crane, "Flow past a stretching plate," Zeitschrift für Angewandte Mathematik und Physik, vol. 21, no. 4, pp. 645-647, 1970.

[5] C. Y. Wang, "The boundary layers due to shear flow over a still fluid," Physics of Fluids A, vol. 4, no. 6, pp. 1304-1306, 1992.

[6] P. S. Gupta and A. S. Gupta, "Heat and mass transfer on a stretching sheet with suction or blowing," The Canadian Journal of Chemical Engineering, vol. 55, pp. 744-746, 1977.

[7] K. R. Rajagopal, T. Y. Na, and A. S. Gupta, "Flow of a viscoelastic fluid over a stretching sheet," Rheologica Acta, vol. 24, no. 2, pp. 213-215, 1984.

[8] B. S. Dandapat and A. S. Gupta, "Flow and heat transfer in a viscoelastic fluid over a stretching sheet," International Journal of Non-Linear Mechanics, vol. 24, no. 3, pp. 215-219, 1989. 
[9] K. R. Rajagopal, T. Y. Na, and A. S. Gupta, "A non-similar boundary layer on a stretching sheet in a non-Newtonian fluid with uniform free stream," Journal of Mathematical and Physical Sciences, vol. 21, pp. 189-200, 1987.

[10] P. D. Weidman, D. G. Kubitschek, and S. N. Brown, "Boundary layer similarity flow driven by power-law shear," Acta Mechanica, vol. 120, pp. 199-215, 1997.

[11] E. Magyari, B. Keller, and I. Pop, "Boundary-layer similarity flows driven by a power-law shear over a permeable plane surface," Acta Mechanica, vol. 163, no. 3-4, pp. 139-146, 2003.

[12] G. E. Cossali, "Similarity solutions of energy and momentum boundary layer equations for a power-law shear driven flow over a semi-infinite flat plate," European Journal of MechanicsB/Fluids, vol. 25, no. 1, pp. 18-32, 2006.

[13] E. Magyari and P. D. Weidman, "Heat transfer on a plate beneath an external uniform shear flow," International Journal of Thermal Sciences, vol. 45, no. 2, pp. 110-115, 2006.

[14] P. D. Weidman, A. M. J. Davis, and D. G. Kubitschek, "Crocco variable formulation for uniform shear flow over a stretching surface with transpiration: multiple solutions and stability," Zeitschrift für Angewandte Mathematik und Physik, vol. 58, no. 2, pp. 313-332, 2008.

[15] T. Fang, "Flow and heat transfer characteristics of the boundary layers over a stretching surface with a uniform-shear free stream," International Journal of Heat and Mass Transfer, vol. 51, no. 9-10, pp. 2199-2213, 2008.

[16] H. Xu, "Homotopy analysis of a self-similar boundary-flow driven by a power-law shear," Archive of Applied Mechanics, vol. 78, no. 4, pp. 311-320, 2008.

[17] F. Aman, A. Ishak, and I. Pop, "Heat transfer at a stretching/shrinking surface beneath an external uniform shear flow with a convective boundary condition," Sains Malaysiana, vol. 40, no. 12, pp. 1369-1374, 2011.

[18] P. G. Drazin and N. Riley, The Navier-Stokes Equations: A Classification of Flows and Exact Solutions, Cambridge University Press, Cambridge, UK, 2006.

[19] J. H. Merkin, "Mixed convection boundary layer flow on a vertical surface in a saturated porous medium," Journal of Engineering Mathematics, vol. 14, no. 4, pp. 301-313, 1980.

[20] T. R. Mahapatra, S. K. Nandy, and A. S. Gupta, "Dual solution of MHD stagnation point flow towards a stretching surface," Engineering, vol. 2, pp. 299-305, 2010. 

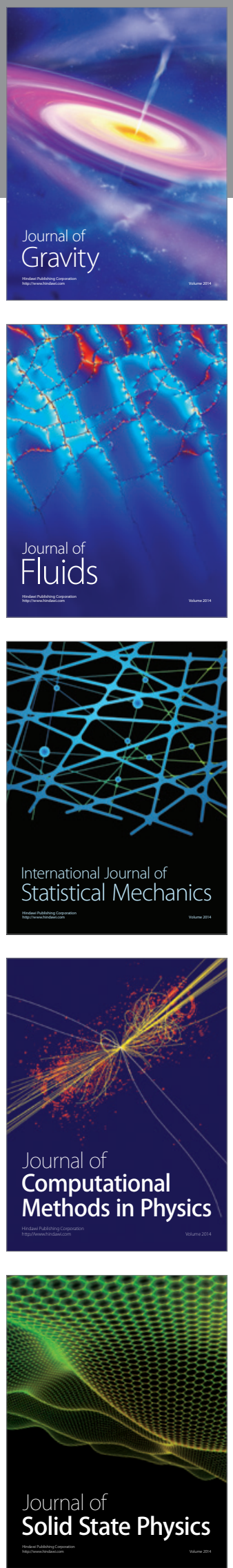

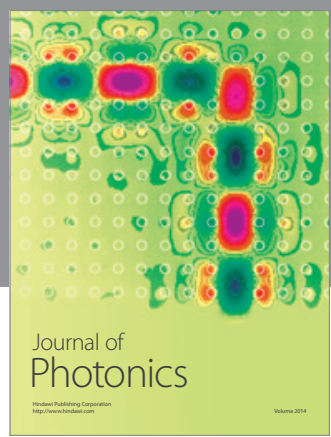

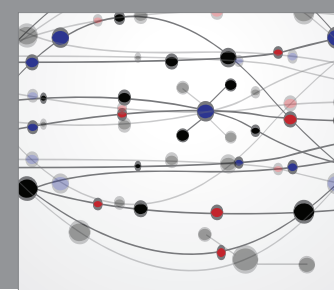

The Scientific World Journal

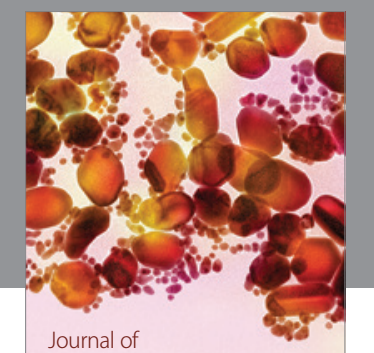

Soft Matter
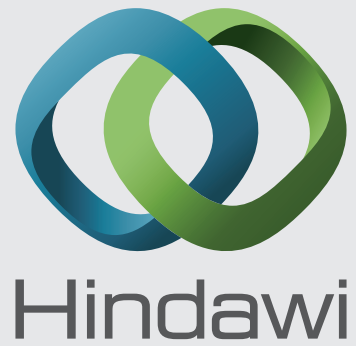

Submit your manuscripts at

http://www.hindawi.com
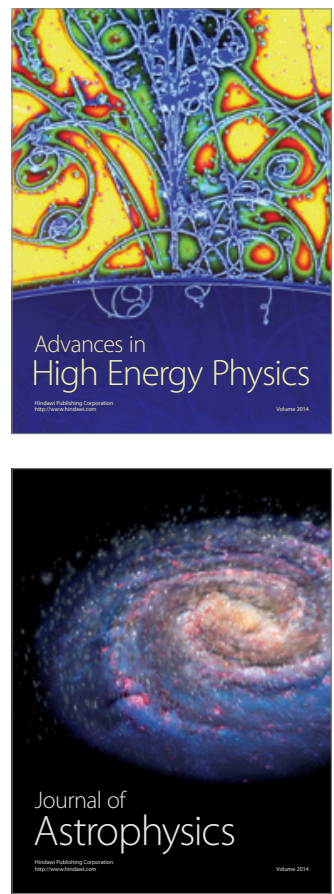
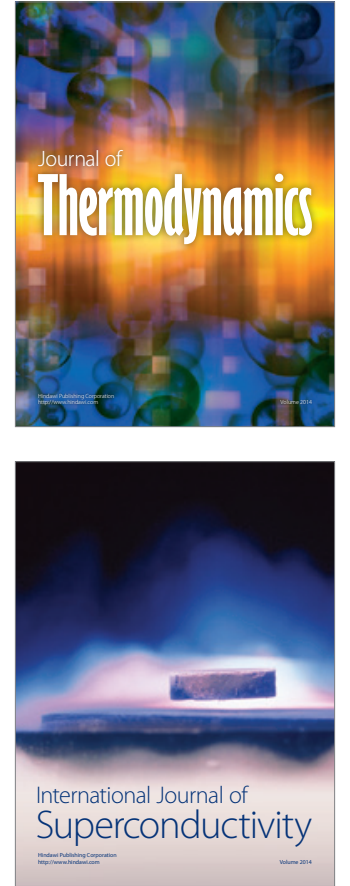
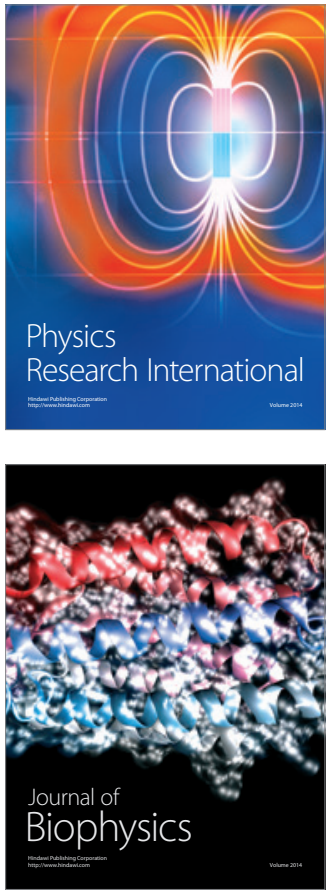
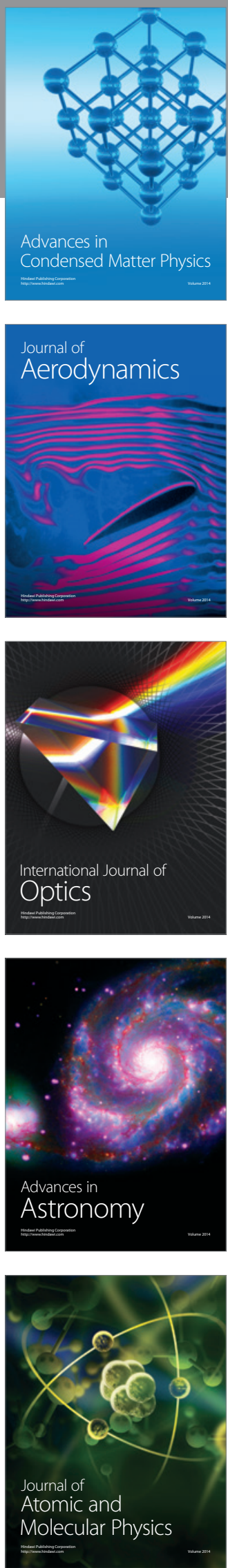